\title{
PLURIPARIDADE NAS ÉGUAS: RELAÇÃO COM CARACTERÍSTICAS MATERNAS, PLACENTÁRIAS E NEONATAIS
}

\section{PLURIPARITY IN MARES: RELATIONSHIP WITH MATERNAL, PLACENTAL, AND NEONATAL CHARACTERISTICS}

\author{
Elena Carolina Serrano-Recalde ${ }^{1}$ \\ Juliana Cristina Queiroz-Silva ${ }^{1}$ \\ Carina de Fatima Guimarães ${ }^{1}$ \\ Mariana Matera Veras ${ }^{2}$ \\ Roberta Galvano Barbosa ${ }^{1}$ \\ Maria Augusta Alonso ${ }^{1}$ \\ Claudia Barbosa Fernandes ${ }^{1 *}$
}

${ }^{1}$ Faculdade de Medicina Veterinária e Zootecnia, Universidade de São Paulo, São Paulo, SP, Brasil. ${ }^{2}$ Faculdade de Medicina, Universidade de São Paulo, SP, Brasil.

*Autora para correspondência - fernandescb@usp.br

\section{Resumo}

A placenta é o órgão de ligação entre a égua e o potro e é um dos principais responsáveis pelo desenvolvimento fetal e pelas características morfométricas do neonato. Essa função se torna cada vez mais importante porque os estudos indicam que as características físicas dos potros predizem o seu desenvolvimento na vida adulta. Desta forma, o objetivo do presente estudo foi correlacionar número de partos (1-5) e características maternas de éguas com as características placentárias e o tamanho do potro. As éguas foram categorizadas em grupos de acordo com o número de partos sendo G1: 1 parto $(n=4) ; G 2: 2$ partos $(n=6) ; G 3: 3$ partos $(n=7) ; G 4: 4$ partos $(n=5)$; e G5: 5 partos $(\mathrm{n}=3)$. Como principais resultados obtidos, o perímetro torácico e o peso da égua pré- e pós-parto influenciaram positivamente peso $(\mathrm{p}=0,004 / \mathrm{R}=0,51 ; \mathrm{p}=0,002 / \mathrm{R}=0,55 ; \mathrm{p}=0,01 / \mathrm{R}=0,43)$, altura $(\mathrm{p}=0,0005 / \mathrm{R}=0,60 ; \mathrm{p}=0,001 / \mathrm{R}=0,57 ; \mathrm{p}=0,005 / \mathrm{R}=0,50)$ e perímetro torácico $(\mathrm{p}=0,0001 / \mathrm{R}=$ $0,65 ; \mathrm{p} \leq 0,0001 / \mathrm{R}=0,71 ; \mathrm{p}=0,0002 / \mathrm{R}=0,64)$ dos potros ao nascimento. Éguas com maior peso corporal no pré-parto apresentaram maior peso placentário $(\mathrm{p}=0,01 / \mathrm{R}=0,45)$ e pariram potros mais pesados $(\mathrm{p}=0,003 / \mathrm{R}=0,52)$ com maior perímetro torácico $(\mathrm{p}=0,01 / \mathrm{R}=0,45)$. Os neonatos de éguas do G4 foram os mais pesados, indicando que provavelmente o maior tamanho uterino em éguas pluríparas permita uma maior cobertura placentária, maior área de contato materno fetal e provavelmente maior aporte de nutrientes ao feto.

Palavras-chave: equino; gestação; membrana corioalantóide; parto.

\section{Abstract}

The placenta connects the mare and the foal and it is one of the main organs responsible for fetal 
development and newborn's morphometric characteristics. This function becomes even more important because a number of studies indicates that foals physical characteristics are able to predict their development in adult life. Therefore, the aim of this study was to correlate mares parity (1-5) and maternal characteristics with placental characteristics as well as foals size. Mares were categorized according to parity in G1: 1 parturation $(n=4) ; G 2: 2$ parturations $(n=6) ; G 3: 3$ parturations $(n=7)$; G4: 4 parturations $(n=5)$; and G5: 5 parturations $(n=3)$. Results indicate that the mares pre- and post-parturition thoracic perimeter and weight had a positive correlation on foals weight $(\mathrm{p}=0.004 / \mathrm{R}=0.51 ; \mathrm{p}=0.002 / \mathrm{R}=0.55 ; \mathrm{p}=0.01 / \mathrm{R}=0.43)$, height $(\mathrm{p}=0.0005 / \mathrm{R}=0.60$; $\mathrm{p}=0.001 / \mathrm{R}=0.57 ; \mathrm{p}=0.005 / \mathrm{R}=0,50)$, and thoracic perimeter $(\mathrm{p}=0.0001 / \mathrm{R}=0.65 ; \mathrm{p} \leq 0.0001 / \mathrm{R}=$ $0.71 ; \mathrm{p}=0.0002 / \mathrm{R}=0.64)$ at birth. Mares that were heavier at pre-partum had greater placental weight $(\mathrm{p}=0.01 / \mathrm{R}=0.45)$ and delivered heavier foals $(\mathrm{p}=0.003 / \mathrm{R}=0.52)$ with greater thoracic perimeter $(\mathrm{p}=0.01 / \mathrm{R}=0.45)$. Foals of mares in $\mathrm{G} 4$ were heavier, probably indicating that the greater uterine size in multiparous mares allows greater placental coverage, greater fetomaternal surface contact and probably greater supply of nutrient to the fetus.

Keywords: chorioallantoic membrane; equine; parturition; pregnancy.

Recebido em: 13 janeiro de 2015

Aceito em: 20 fevereiro de 2017

\section{Introdução}

Diante do desenvolvimento alcançado pela indústria do cavalo, animais que apresentem características genéticas desejáveis e bom desempenho funcional tornam-se uma necessidade do mercado. Partindo deste princípio, o aprimoramento de estudos que correlacionem variáveis maternas e placentárias com as características físicas dos potros ao nascimento, a fim de predizer de forma estratégica o desenvolvimento desses indivíduos em sua vida adulta, assume grande importância. Segundo Allen et al. ${ }^{(1)}$ e Veronesi et al. ${ }^{(2)}$, tais correlações podem ser determinantes no desempenho atlético futuro dos produtos.

O número de partos é um exemplo de variável a ser estabelecida ${ }^{(1,3-5)}$, uma vez que pode estar correlacionada com a função placentária que, por sua vez, interfere no suprimento de nutrientes para o feto e consequentemente no desenvolvimento do mesmo, tendo influência direta sobre o peso e tamanho dos potros ao nascimento ${ }^{(1,4)}$.

Sabe-se que o peso da placenta aumenta progressivamente no decorrer das gestações e o tamanho deste órgão afeta diretamente a capacidade de transferência de nutrientes pela alteração na área de superfície para transporte ${ }^{(6)}$. Assim, deficiências na estrutura e função placentária podem refletir em atrasos na maturidade e crescimento do feto ${ }^{(4)}$, bem como distúrbios severos podem levar à morte fetal $^{(7)}$. Considerando que o peso do potro ao nascimento está positivamente correlacionado com a área total e microscópica do alantocórion ${ }^{(1,4,8,9)}$, as alterações mencionadas anteriormente resultam em neonatos com escore de condição corporal abaixo do esperado, podendo representar um comprometimento do potencial atlético e rendimento futuro do indivíduo ${ }^{(2)}$. Em humanos, estudos demonstraram uma forte associação entre baixo peso ao nascimento e o desenvolvimento de doenças metabólicas em adultos ${ }^{(10,11)}$. 
Desta forma, o objetivo do presente trabalho foi correlacionar as características maternas e o número de partos de éguas com o tamanho do neonato (peso, altura e perímetro torácico dos potros) e características placentárias (peso da membrana alantocoriônica, comprimento do cordão umbilical e densidade microcotiledonária).

\section{Material e Métodos}

Foram utilizadas vinte e cinco éguas prenhes de potros da raça Quarto de Milha alocadas em um haras localizado em Brotas, no Estado de São Paulo - Brasil, durante a estação de parição de 2011. As éguas foram categorizadas em grupos de acordo com o número de partos, considerando G1: 1 parto $(\mathrm{n}=4)$, com idade média de 11.00 \pm 2.00 anos; $\mathrm{G} 2: 2$ partos $(\mathrm{n}=6)$ com idade média de

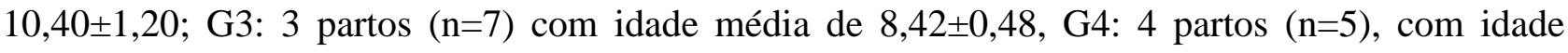

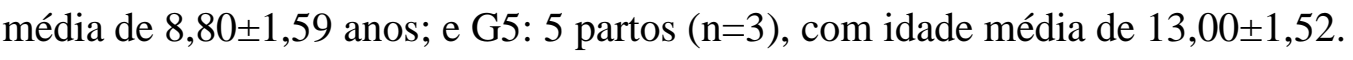

O peso das éguas (pré e pós-parto imediato) e o peso dos neonatos às 12 horas após o nascimento foram obtidos em quilogramas $(\mathrm{Kg})$ por meio de balança digital (Digitron ${ }^{\circledR}$, Digi-tron, Agudos, SPBrasil). A altura das éguas, assim como também o perímetro torácico de éguas e potros foram mensurados em centímetros $(\mathrm{cm})$ utilizando-se fita métrica. Enquanto a medição de altura dos potros foi realizada por meio de hipômetro às 12 horas após o nascimento. A idade, o número de partos e o tempo de gestação dos animais foram adquiridos pela revisão das fichas gestacionais das respectivas fêmeas.

O tempo de delivramento foi cronometrado e os anexos fetais foram recolhidos imediatamente após a expulsão para a aferição do peso total $(\mathrm{Kg})$ em balança digital (Digitron®, Digi-tron, Agudos, SPBrasil). O comprimento do cordão umbilical $(\mathrm{cm})$ foi mensurado por meio de fita métrica somandose o lado placentário e o lado neonatal. Após a avaliação macroscópica, as placentas foram

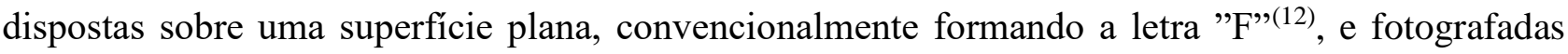
com escala para posteriores aferições de sua área de superfície.

Foram coletados fragmentos em duplicata de $3 \mathrm{~cm}^{2}$ da membrana corioalantóide nas regiões do corpo do útero $(\mathrm{CP})$, corno uterino gestante $(\mathrm{CG})$ e corno uterino não gestante $(\mathrm{CnG})$ em distâncias pré-definidas por meio de fita métrica $(\mathrm{cm})$. Para a coleta do fragmento correspondente ao corpo uterino, foi considerada uma distância de $35 \mathrm{~cm}$ a partir da estrela cervical, para o corno gestante e corno não gestante, foi considerada uma distância de $35 \mathrm{~cm}$ a partir do ápice do respectivo corno. Os cortes foram fixados em formalina a $10 \%$ à temperatura ambiente.

Fragmentos do tecido corioalantóide foram diafanizados em diferentes concentrações de álcool e xilol e incluídos em duplicata em blocos de parafina. Cortes de $5 \mu \mathrm{m}$ foram obtidos, montados sobre lâminas histológicas, desparafinizados, hidratados e corados pelo método de hematoxilina-eosina (HE) segundo a técnica descrita por Allen ${ }^{(13)}$.

A avaliação da densidade microcotiledonária foi realizada por meio da seleção randômica de cinco campos em cada lâmina das três regiões coletadas. As lâminas foram posicionadas no microscópio óptico (Eclipse E200®, Nikon) e as imagens foram feitas com uma câmera fotográfica (SC30®, Olympus) acoplada ao microscópio, em aumento de 2,5 vezes. 
Após a realização das micrografias, foi contado o número de microcotilédones em cada imagem. Para tal contagem utilizou-se o programa ImageJ®, com a inserção de um retângulo, sempre na mesma posição; assim, apenas os microcotilédones contidos no retângulo destacado participavam da contagem (Figura 1). Para cada égua foi feita a média do número de microcotilédones de cada região (corno gestante, corno não gestante e corpo do útero) da membrana corioalantóide.

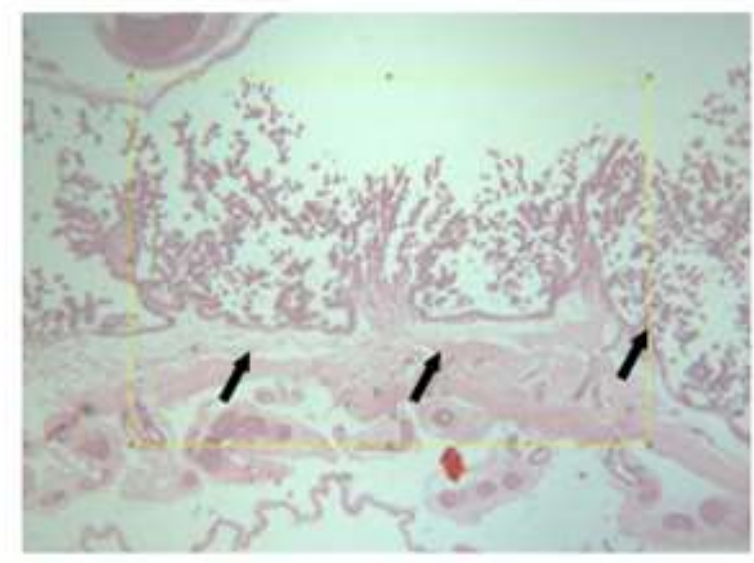

Figura 1. Micrografia da membrana corioalantóide a termo na região do corno uterino gestante de éguas. Presença de um retângulo que auxilia na contagem dos microcotilédones. Nessa foto, observam-se três microcotilédones (setas pretas). Aumento de $2,5 \mathrm{x}$, coloração hematoxilina-eosina.

Os dados obtidos foram analisados por meio do programa SAS System for Windows 9.3 (SAS, 2000). O aplicativo Guided Data Analisys foi utilizado na avaliação da normalidade dos resíduos (distribuição normal) e homogeneidade das variâncias. Caso não obedecessem a estas premissas, foram transformados (logaritmo na base 10 - Log10X; Raiz quadrada - RQ X; Quadrado - X2) e se a normalidade não fosse obtida, empregava-se, então, o procedimento NPAR1WAY de análise de variância não paramétrica. $\mathrm{Na}$ avaliação do efeito do tempo e do tratamento nas variáveis resposta, foram utilizados os testes Duncan (comparações múltiplas entre os grupos de parto - G1, G2, G3, G4 e G5) e t de Student (sexo do potro) para variáveis paramétricas e os testes Kruskal Wallis e Wilcoxon para variáveis não paramétricas. Foram empregadas as médias e seus respectivos errospadrão (média \pm erro-padrão da média) dos dados originais para descrição dos resultados. $\mathrm{O}$ nível de significância utilizado para rejeitar H0 (hipótese de nulidade) foi de 5\%, isto é, para um nível de significância menor que 0,05 , considerou-se que ocorreram diferenças estatísticas entre os grupos estudados. As variáveis resposta foram submetidas à análise de correlação de Pearson e Spearman, para variáveis paramétricas e não paramétricas, respectivamente. A regressão linear (PROC REG) foi utilizada para a avaliação da relação quantitativa entre as variáveis relacionadas à égua e aquelas relacionadas aos potros.

O presente trabalho foi aprovado pela Comissão de Ética no uso de animais da Faculdade de Medicina Veterinária e Zootecnia da Universidade de São Paulo protocolado sob o nº 2049/2010. 


\section{Resultados}

Os valores médios, mínimos e máximos obtidos nos parâmetros avaliados encontram-se descritos na Tabela 1.

Tabela 1. Valores da média \pm erro-padrão, valores mínimo e máximo para os parâmetros maternos, placentários e neonatais, coletados em 25 partos de éguas prenhes de potros da raça Quarto de Milha. Brotas, São Paulo - Brasil, 2011

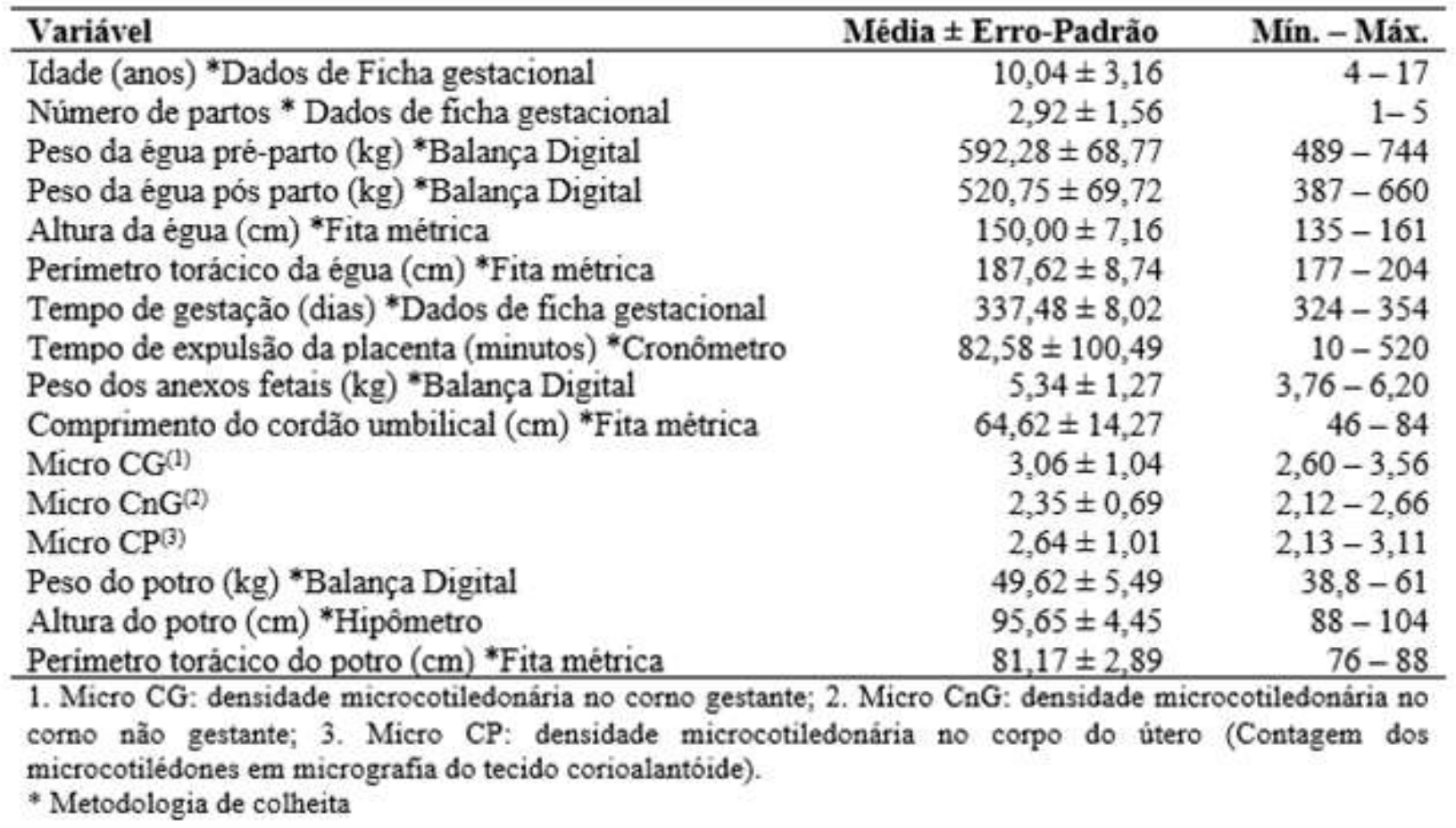

$\mathrm{O}$ perímetro torácico e o peso da égua no pré- e pós-parto influenciaram positivamente o peso $(\mathrm{p}=0,004$ e $\mathrm{R}=0,51 ; \mathrm{p}=0,002$ e $\mathrm{R}=0,55 ; \mathrm{p}=0,01$ e $\mathrm{R}=0,43)$, a altura $(\mathrm{p}=0,0005$ e $\mathrm{R}=0,60 ; \mathrm{p}=0,001$ e $\mathrm{R}=0,57 ; \mathrm{p}=0,005$ e $\mathrm{R}=0,50)$ e o perímetro torácico $(\mathrm{p}=0,0001$ e $\mathrm{R}=0,65 ; \mathrm{p}=\leq 0,0001$ e $\mathrm{R}=0,71$; $\mathrm{p}=0,0002$ e $\mathrm{R}=0,64)$ dos potros ao nascimento.

As fêmeas mais pesadas no período pré-parto apresentaram placentas mais pesadas $(\mathrm{p}=0,01 \mathrm{e}$ $\mathrm{R}=0,45)$ e cordões umbilicais mais longos ( $\mathrm{p}=0,03$ e $\mathrm{R}=0,40$ ). Da mesma forma, as éguas com placentas mais pesadas tiveram cordões umbilicais mais longos $(p=0,001$ e $R=0,56)$ e pariram potros mais pesados ( $\mathrm{p}=0,003$ e $\mathrm{R}=0,52)$ e com maior perímetro torácico $(\mathrm{p}=0,01$ e $\mathrm{R}=0,45)$.

Quando os animais foram divididos nos grupos experimentais, a análise dos dados demonstrou que o peso dos anexos fetais $(7,36 \pm 1,47 \mathrm{~kg})$ e o comprimento do cordão umbilical $(91,66 \pm 9,66 \mathrm{~cm})$ foram superiores nas éguas do G5 quando comparadas aos demais grupos (Tabela 2). Este foi o segundo grupo que gestou os potros mais pesados, juntamente com G1, G2 e G3. Além disso, para cada kg extra de peso da égua no pré- e pós-parto, o peso, a altura e o perímetro torácico dos potros ao nascimento obtiveram um incremento na ordem de $0,04 \mathrm{~kg}$ e $0,02 \mathrm{~kg} ; 0,04 \mathrm{~cm}$ e $0,03 \mathrm{~cm}$; e 0,02 cm e $0,02 \mathrm{~cm}$, respectivamente. 
Tabela 2. Valores da média \pm erro-padrão dos parâmetros avaliados que apresentaram diferença estatística entre os grupos estabelecidos (G1: 1 parto; G2: 2 partos; G3: 3 partos; G4: 4 partos; e G5: 5 partos), em 25 partos de éguas prenhes de potros da raça Quarto de Milha. Brotas, São Paulo - Brasil, 2011

\begin{tabular}{|c|c|c|c|c|c|}
\hline Variáveis & $\begin{array}{c}\mathbf{G l} \\
(\mathbf{n}=4)\end{array}$ & $\begin{array}{c}\mathbf{G 2} \\
(\mathbf{n}=6)\end{array}$ & $\begin{array}{c}\mathbf{G 3} \\
(\mathrm{n}=7)\end{array}$ & $\begin{array}{c}\mathbf{G 4} \\
(\mathrm{n}=5)\end{array}$ & $\begin{array}{c}\text { G5 } \\
(\mathbf{n}=3)\end{array}$ \\
\hline & \multicolumn{5}{|c|}{ Média \pm Erro-padrão } \\
\hline Idade (anos) & $11,00 \pm 2,00^{2 b}$ & $10,40 \pm 1,20^{26}$ & $8,42 \pm 0,48^{b}$ & $8,80 \pm 1,59^{a b}$ & $13,00 \pm 1,52^{a}$ \\
\hline $\begin{array}{l}\text { Peso da égua } \\
\text { pré-parto (kg) }\end{array}$ & $581,75 \pm 49,55^{\circ b}$ & $554,67 \pm 17,47^{\circ}$ & $603,86 \pm 23,35^{26}$ & $593,20 \pm 21,90^{\mathrm{ab}}$ & $678,00 \pm 66,00^{2}$ \\
\hline $\begin{array}{l}\text { Peso da égua } \\
\text { pós parto (kg) }\end{array}$ & $511,25 \pm 49,19$ & $476,67 \pm 21,62^{b}$ & $535,14 \pm 19,35^{\text {ab }}$ & $514,40 \pm 25,77^{\mathrm{ab}}$ & $588,33 \pm 36,79^{\circ}$ \\
\hline $\begin{array}{l}\text { Tempo de } \\
\text { gestação (dias) }\end{array}$ & $330,16 \pm 1,70^{\circ}$ & $334,00 \pm 3,08^{2 b}$ & $340,42 \pm 3,50^{\circ}$ & $338,40 \pm 2,06^{\mathrm{ab}}$ & $341,33 \pm 4,9^{a}$ \\
\hline $\begin{array}{l}\text { Peso dos anexos } \\
\text { fetais }(\mathrm{kg})\end{array}$ & $4,67 \pm 0,18^{b}$ & $5,09 \pm 0,46^{b}$ & $5,13 \pm 0,42^{b}$ & $5,72 \pm 0,16^{6}$ & $7,36 \pm 1,47^{a}$ \\
\hline $\begin{array}{l}\text { Comprimento } \\
\text { do cordão } \\
\text { umbilical }(\mathrm{cm})\end{array}$ & $58,75 \pm 3,54^{b}$ & $66,66 \pm 2,74^{b}$ & $63,71 \pm 5,30^{b}$ & $54,40 \pm 3,64^{b}$ & $91,66 \pm 9,66^{a}$ \\
\hline $\begin{array}{l}\text { Peso do potro } \\
(\mathrm{kg})\end{array}$ & $46,75 \pm 3,09 b$ & $47,70 \pm 0,99^{\circ}$ & $48,68 \pm 1,71^{b}$ & $55,00 \pm 1,30^{a}$ & $51,93 \pm 3,26^{6}$ \\
\hline
\end{tabular}

Letras diferentes na mesma linha representam diferença estatística $(p<0,05)$.

O número de partos foi positivamente correlacionado à duração da gestação $(\mathrm{p}=0,01 ; \mathrm{r}=0,46)$. Nesse sentido, observou-se que os potros nascidos de éguas com gestação mais prolongada foram mais altos ( $\mathrm{p}=0,01$ e $\mathrm{R}=0,46$ ), sendo que, para cada dia a mais de gestação, a altura do potro ao nascimento aumentou em $0,27 \mathrm{~cm}$. Ainda assim, o peso do neonato foi fortemente correlacionado com sua altura ( $\mathrm{p}=\leq 0,0001$ e $\mathrm{R}=0,72)$ e perímetro torácico $(\mathrm{p}=\leq 0,0001$ e $\mathrm{R}=0,87)$ ao nascimento.

Não houve associação significativa entre o tempo de gestação e o peso da placenta ( $\mathrm{p}=0,98$ e $\mathrm{R}=-$ $0,003$ ), o peso ( $\mathrm{p}=0,32$ e $\mathrm{R}=0,19)$ e o perímetro torácico ( $\mathrm{p}=0,19$ e $\mathrm{R}=0,24)$ do potro ao nascimento.

$\mathrm{Na}$ análise de regressão linear para cada parto, o peso da placenta e do potro ao nascimento apresentaram um incremento na ordem de $0,3 \mathrm{~kg}$ e 1,69 $\mathrm{kg}$, respectivamente. Para cada $\mathrm{kg}$ extra da massa total placentária, o peso do potro ao nascimento aumentou $2,97 \mathrm{~kg}$.

$\mathrm{O}$ peso dos anexos fetais foi positivamente correlacionado com o peso ( $\mathrm{p}=0,003$ e $\mathrm{R}=0,52)$ dos potros ao nascimento. Potros nascidos de anexos fetais mais pesados apresentaram maior perímetro torácico $(\mathrm{p}=0,01$ e $\mathrm{R}=0,45)$.

No presente estudo não foram encontradas diferenças estatísticas significavas entre os grupos na densidade microcotiledonária total na somatória das regiões avaliadas, bem como quando se avaliou separadamente cada zona placentária (Tabela 3). 
Tabela 3. Densidade microcotiledonária (média e erro-padrão) observada nas zonas placentárias (Micro CG: densidade microcotiledonária no corno gestante; Micro CnG: densidade microcotiledonária no corno não gestante; Micro CP: densidade microcotiledonária no corpo uterino) da membrana corioalantóide a termo, de acordo como número de parto (G1: 1 parto; G2: 2 partos; G3: 3 partos; G4: 4 partos; e G5: 5 partos). Brotas, São Paulo, Brasil 2011

\begin{tabular}{lccccc}
\hline & G1 & G2 & G3 & G4 & G5 \\
\hline \multicolumn{5}{c}{ Média \pm Erro-padrão } \\
\hline Micro CG & $3,10 \pm 0,65$ & $3,05 \pm 0,37$ & $3,56 \pm 1,37$ & $2,60 \pm 0,33$ & $3,33 \pm 0,33$ \\
Micro CnG & $2,32 \pm 0,59$ & $2,18 \pm 0,22$ & $2,66 \pm 0,29$ & $2,12 \pm 0,23$ & $2,43 \pm 0,56$ \\
Micro CP & $2,67 \pm 0,43$ & $2,73 \pm 0,42$ & $3,11 \pm 0,56$ & $2,54 \pm 0,40$ & $2,13 \pm 0,29$ \\
\hline
\end{tabular}

Não houve diferença estatística ente os grupos ( $\mathrm{p}>0,05)$.

\section{Discussão}

Este trabalho teve como objetivo correlacionar o número de partos e as características maternas de éguas com as características placentárias e neonatais.

A duração da gestação observada no presente estudo variou entre 324 a 354 dias, com média de $337,48 \pm 8,02$ dias. Valores aproximados foram descritos para a raça Quarto de Milha, em que foi observado tempo gestacional entre 333-343 dias $^{(14)}$ e 339-344 dias ${ }^{(15)}$. Porém, neste estudo não foi encontrada relação entre a idade da fêmea e o tempo de gestação ( $\mathrm{p}=0,52$ e $\mathrm{R}=-0,13)$. Por outro lado, éguas com maior número de partos tiveram maior duração da gestação, e éguas com gestação mais prolongada, consequentemente, pariram potros mais altos.

Corroborando a informação anterior, as éguas do G5 tiveram anexos fetais mais pesados $(7,36 \pm 1,47$ $\mathrm{kg}$ ), quando comparados aos de todos os outros grupos estudados. Da mesma forma, os potros neonatos nascidos do grupo de éguas com quatro partos apresentaram maior peso. Assim, apesar de a literatura sugerir que éguas mais velhas e pluríparas tendem a apresentar uma diminuição na eficiência placentária devido à menor área de troca de gases e nutrientes em resposta a degenerações ou disfunções das glândulas endometriais ${ }^{(9)} \mathrm{e}$, consequentemente, à pluriparidade, levando a um menor crescimento fetal intrauterino e prolongamento da gestação ${ }^{(16)}$, no presente estudo, tanto uma gestação mais prolongada como o número de parições da égua influenciaram diretamente e de forma positiva o crescimento intrauterino do potro. Os resultados também indicam não haver alteração significativa da densidade microcotiledonária de éguas pluríparas com maior média de idade $(13,00 \pm 1,52)$, em relação aos outros grupos estudados. Essa diferença entre os achados da literatura e os apresentados aqui podem ser explicada pela baixa média de idade dos animais pertencentes aos G4 e G5, o que provavelmente correspondeu a animais com boa saúde uterina. 
Em contrapartida, apesar da não diferença estatística, os potros dos grupos de 1 a 3 partos foram numericamente mais leves ao nascimento, o que corrobora em parte o descrito na literatura ${ }^{(4,17)}$, em que potros provenientes de éguas primíparas apresentaram peso inferior quando comparados aos nascidos de éguas pluríparas. Avaliações obstétricas em mulheres demonstraram que bebês de gestantes pluríparas a partir de 32 semanas de gestação são mais pesados do que os bebês de gestantes primíparas. Assim como as placentas de pluríparas são mais pesadas do que primíparas a partir de 25 semanas de gestação ${ }^{(18)}$. Bhuvanakumar \& Satchidanandam ${ }^{(19)}$ afirmaram que uma provável razão para que éguas primíparas gestem potros mais leves é o fato de que nestas fêmeas ocorre uma restrição do espaço intrauterino para o desenvolvimento fetal, devido a uma redução na capacidade de distensão da parede uterina, o que parece confirmar os resultados apresentados neste trabalho.

Wilsher \& Allen ${ }^{(4)}$ identificaram que éguas nulíparas e pluríparas com idade avançada apresentaram uma menor área macroscópica, massa e volume do corioalantóide, assim como uma redução na área de superfície e de contato dos microcotilédones, quando comparadas a éguas jovens pluríparas ${ }^{(4)}$. No entanto, no presente estudo, não foram encontradas diferenças estatísticas significativas quanto à densidade microcotiledonária nos grupos de paridade e regiões da membrana corioalantóide analisados.

Em equinos, a relação entre idade e paridade com o peso do potro já foi descrita por Wilsher \& Allen $^{(4)}$, Naverrete et al. ${ }^{(20)}$ e Elliott et al. ${ }^{(17)}$. Esses autores relataram que a paridade apresentou maior influência no peso dos potros ao nascimento do que a idade materna, achados similares aos deste estudo. Dessa forma, o remodelamento da estrutura vascular placentária, devido às gestações anteriores e um ambiente uterino mais favorável para o desenvolvimento e função placentária para as próximas gestações, ${ }^{(18)}$ pode ser o fator determinante para o estabelecimento da maior eficiência placentária. Para Wilsher \& Allen ${ }^{(4)}$, as éguas atingem esse platô de eficiência entre 10 a 15 anos de idade. Essas descrições podem justificar a ausência de diferenças nas estruturas microscópicas avaliadas e também justificar o nascimento de potros mais pesados no G4, constituído de éguas jovens e pluríparas.

No presente estudo, as éguas com maior perímetro torácico e maior peso no pré- e pós-parto produziram potros mais pesados, de maior altura e perímetro torácico. Ao mesmo tempo em que fêmeas mais pesadas no período pré-parto apresentaram placentas mais pesadas e cordões umbilicais mais longos. Dados similares foram reportados para éguas Holsteiner ${ }^{(20)}$, nos quais o peso materno influenciou positivamente o peso dos potros ao nascimento. Segundo Wilsher \& Allen ${ }^{(5)}$, o tamanho uterino é diretamente relacionado ao porte materno e, dessa forma, pode influenciar o tamanho/peso da placenta, a área de placentação e, consequentemente, o crescimento fetal.

O tamanho da fêmea é, portanto, outro fator de grande importância no que se refere à escolha da égua. Experimentos prévios ${ }^{(1,21)}$ têm demonstrado que, em equinos, o potencial genético normal de crescimento fetal de uma raça pode ser tanto aumentado como restringido por meio da variação do tamanho materno. No entanto, os mecanismos pelos quais os genótipos materno, fetal e fatores epigenéticos que controlam o desenvolvimento placentário e neonatal ainda permanecem desconhecidos.

O fenômeno do aumento do peso placentário e do recém-nascido e a relação destes com a paridade materna tem sido reportado em humanos ${ }^{(22,23)}$, equinos ${ }^{(4,17,20)}$ e outros mamíferos ${ }^{(24-}{ }^{27)}$. Nesse 
trabalho, o peso da placenta variou entre 3,76 a $6,20 \mathrm{~kg}$ (média 5,34 $\pm 1,27 \mathrm{~kg}$ ) e o comprimento do cordão umbilical mediu entre 46 a $84 \mathrm{~cm}$. Dados similares foram reportados para éguas da raça Puro Sangue Inglês (PSI) ${ }^{(28)}$ e Standarbred ${ }^{(29)}$ nos quais o peso das membranas fetais variou entre 4 a $8,4 \mathrm{~kg}$, e 2,7 a $6,1 \mathrm{~kg}$ e o comprimento do cordão umbilical de 36 a $83 \mathrm{~cm}$, e 29 a $87 \mathrm{~cm}$ respectivamente.

O peso dos anexos fetais foi positivamente correlacionado com o peso e o perímetro torácico dos potros ao nascimento, concordando com estudos prévios $^{(1,4,8,9,17,20,30,31)}$. O peso médio total dos anexos fetais correspondeu a $10,76 \%$ do peso médio dos recém-nascidos. Valor muito próximo foi constatado por Whitwell \& Jeffcott ${ }^{(28)}$, para a raça PSI, no qual a relação entre o peso da placenta e o peso dos potros ao nascimento foi de $11 \%$, observando uma relação linear entre essas variáveis.

Elliott et al. ${ }^{(17)}$ observaram que para cada quilograma de aumento no peso placentário ocorreu um incremento de 4,5 kg no peso do potro, assim como para cada parto subsequente, o peso do recémnascido obteve um aumento na ordem de $0,6 \mathrm{~kg}$ e o peso dos anexos fetais de 0,06 $\mathrm{kg}$. Dados similares foram obtidos neste estudo, sendo que, para cada parto, o peso da placenta e do potro ao nascimento apresentaram um incremento na magnitude de $0,3 \mathrm{~kg}$ e $1,69 \mathrm{~kg}$, respectivamente. Para cada quilograma extra da massa total placentária, o peso do potro ao nascimento obteve um aumento de $2,97 \mathrm{~kg}$.

\section{Conclusões}

Apesar da densidade microcotiledonária similar observada entre os grupos deste estudo, sugere-se, baseado na literatura e nos resultados encontrados, que o maior tamanho uterino em éguas pluríparas permite uma maior cobertura placentária, uma maior área de contato materno fetal e provavelmente maior aporte de nutrientes ao potro, resultando em neonatos com maior peso, altura e perímetro torácico. Outras pesquisas devem ser realizadas para determinar se essas diferenças perduram na vida adulta e interferem na saúde e desempenho atlético dos animais.

\section{Agradecimentos}

À FAPESP (Processo no: 2010/18474-0) e ao CNPq (Processo 470664/2013-4).

\section{Referências}

1. Allen WR, Wilsher S, Turnbull C, Stewart F, Ousey J, Rossdale PD, et al. Influence of maternal size on placental, fetal and postnatal growth in the horse. Development in utero. Reproduction. 2002;123(3):445453a.

2. Veronesi MC, Villani M, Wilsher S, Contri A, Carluccio A. A comparative stereological study of the term placenta in the donkey, pony and Thoroughbred. Theriogenology. 2010;74(4):627-631

3. Tischner M. Development of Polish-pony foals born after embryo transfer to large mares. Journal of 
Reproduction and Fertility Supplement. 1987;35:705-709.

4. Wilsher S, Allen WR. The effects of maternal age and parity on placental and fetal development in the mare. Equine Veterinary Journal. 2003;35(5):476-483.

5. Wilsher S, Allen WR. Factors influencing placental development and function in the mare. Equine Veterinary Journal. 2012; v. 44(Suppl 41):113-119.

6. Fowden AL, Ward JW, Wooding FBP, Forhead AJ, Constancia M. Programming placental nutrient transfer capacity. Journal of Physiology. 2006;572(1):5-15.

7. Whitwell KE. Investigations into fetal and neonatal losses in the horse. Veterinary Clinics of North America: Large Animal Practice. 1980; 2:313-331.

8. Cottrill CM, Jeffers-Lo J, Ousey JC, Mcgladdery AJ, Ricketts SW, Silver M, et al. The placenta as a determinant of fetal wellbeing in normal and abnormal pregnancies. Journal of Reproduction and Fertility Suppl.,1991;44:591-601.

9. Bracher V, Mathias S, Allen WR. Influence of chronic degenerative endometritis (endometrosis) on placental development in the mare. Equine Veterinary Journal, 1996;28(3):180-188.

10. Barker DJP. Intrauterine programming of adult disease. Molecular Medicine Today. 1995;1(9):418-423

11. Barker DJP. Chronic disease in humans originates in the intra-uterine environment. Animal Reproduction Science. 2006;94:343-354.

12. Ashbury AC, Leblanc MM. The placenta. In: A.O. McKinnon and J.L. Voss, editors. Equine Reproduction. Philadelphia: Lea\& Febiger; 1993. p.509-516.

13. Allen TC. Hematoxilin and eosin. In: Prophet EB, Mills B, Arrington JB, Sobin LH. (ED). Laboratory Methods in Histotechnology - Armed Forces Institute of Pathology. Washington: American Registry of Pathology, 1994, p.53-57.

14. Pool-Anderson K, Raub RH, Warren JA. Maternal influences on growth and development of full-sibling foals. Journal of Animal Science. 1994;72:1661-1666.

15. Guay KA, Brady HA, Allen VG, Pond KR, Wester DB, Janecka LA, et al. Matua bromegrass hay for mares in gestation and lactation. Journal of Animal Science. 2002;80:2960-2966.

16. Morel MCGD, Newcombe JR, Holland SJ. Factors affecting gestation length in the Thoroughbred mare. Animal Reproduction Science. 2002;74:175-185.

17. Elliott C, Morton J, Chopin J. Factors affecting foal birth weight in Thoroughbred horses. Theriogenology. 2009;71(4):683-689.

18. Kloosterman GJ. On intrauterine growth: the significance of prenatal care. International Journal of Gynecology and Obstetrics. 1970;8:895-912.

19. Bhuvanakumar CK, Satchidanandam V. Effect of parity on the birthweight of foals in Thoroughbreds. Centaur. 1989;6:43-45.

20. Navarrete KR, Muñoz AL, Saravia FR, Quezada M. Determinación del peso normal Del alanto-corion em yeguas Holsteiner. Avances en Ciencias Veterinarias. 2005;20:61-66.

21. Tischner M. Embryo recovery from Polish-pony mares and preliminary observations on foal size after transfer of embryos to large mares. Equine Veterinary Journal Supplement. 1985;3:96-98

22. Wilcox MA, Chang AMZ, Johnson IR. The effects of parity on birthweight using successive pregnancies. Acta Obstetricia et Gynecologica Scandinavica. 1996;75:459-463.

23. Khong TY, Adema ED, Erwich JHM. On anatomical basis for the increase in birth weight in second and subsequent born children. Placenta. 2003;24:348-353.

24. Burris MJ, Blunn CT. Some factors affecting gestation length and birthweight of beef cattle. Journal of Animal Science.1952;11:34-43.

25. Broadhurst PL, Jinks JL. Parity as a determinant of birthweight in the rhesus monkey. Folia Primatology. 
1965;3:201-204.

26. Mccarthy JC. Genetic and environmental control of foetal and placental growth in the mouse. Animal Production.1965;7:347-351.

27. Clarke L, Yakubu DP, Symonds ME. Influence of maternal bodyweight on size, conformation and survival of newborn lambs. Reproduction, Fertility and Development.1997;9:509-514.

28. Whitwell KE.; Jeffcott LB. Morphological studies on the fetal membranes of the normal singleton foal at term. Research in Veterinary Science. 1975;19:44-55.

29. Whitehead AE, Chenier TS, Foster RA. Placental characteristics of Standardbred mares. Proceedings of the American Association of Equine Practitioner. 2006;51:215-220.

30. Allen WR, Wilsher S, Stewart F, Ousey J, Fowden A. The Influence of maternal size on placental, fetal and postnatal growth in the horse: II Endocrinology of pregnancy. Journal of EndocrinologY. 2002;172:237$246 \mathrm{~b}$

31. Fernandes CB, Meirelles MG, Guimarães CF, Nichi M, Affonso FJ, Fonte JS, et al. Which paternal, maternal and placental parameters influence foal size and vitality? Journal of Equine Veterinary Science. 2014;34:225-227. 